\title{
The Generic Contact of Convex Bodies with Circumscribed Homothets of a Convex Surface
}

\author{
Andreana Zucco \\ Dipartimento di Matematica, Università di Torino, \\ Via Carlo Alberto 10, 10123 Torino, Italia
}

\begin{abstract}
Let $S_{0}$ be a convex surface in $d$-dimensional Euclidean space $E^{d}$. Then, if $S_{0}$ is smooth and strictly convex, we prove that the typical convex body touches the convex suface circumscribed about it and homothetic to $S_{0}$ at precisely $d+1$ points.
\end{abstract}

\section{Introduction}

Let $S_{0}$ be a convex surface in $d$-dimensional Euclidean space $E^{d}$. We assume $S_{0}$ to be smooth and strictly convex, which means that $S_{0}$ is of class $\mathscr{C}^{1}$ and includes no line segment. Let $\mathscr{S}$ be the set of all surfaces directly homothetic to $S_{0}$.

For a given convex body $C$, we say that the surface $S(C) \in \mathscr{S}$, whose convex hull conv $S$ includes $C$ and has minimal volume, known to be unique, is $\mathscr{S}$ circumscribed about $C$.

By a theorem of Zamfirescu [4], most convex bodies in the sense of Baire categories touch their circumscribed spheres at precisely $d+1$ points. Here we strengthen this result to any family of $\mathscr{P}$-circumscribed surfaces, i.e., with $S_{0}$ not necessarily a sphere, but any smooth strictly convex surface. Moreover, we show that these $d+1$ points form an irreducible set (see the definition below).

Other applications of the Baire category theorem in convexity may be found in [3] and [6] and the surveys [2] and [5].

\section{Notations and Definitions}

We denote by $\mathscr{C}$ the space of convex bodies in $E^{d}$, i.e., the space of all compact convex subsets of $E^{d}$ with nonempty interior endowed with the Hausdorff metric. It is known that $\mathscr{C}$ is a Baire space. 
We say that a property holds for most elements of some Baire space, or that it is typical, if the set of those elements which do not have this property is of the first Baire category, i.e., a countable union of nowhere dense sets.

The abbreviations bd, int, card, and conv stand for boundary, interior, cardinality, and convex hull.

Since we use the Gauss map, we recall its definition here. Let $S \subset E^{d}$ be a convex surface. Given a point $x$ of $S$, define its normal image $n(x)$ to be the subset of the $(d-1)$-dimensional unit sphere $S^{d-1}$ consisting of the points $y$ such that the supporting hyperplane to $S^{d-1}$ at $y$ is parallel to a supporting hyperplane of $S$ at $x$ (with $S^{d-1}$ and $S$ contained in the corresponding half-spaces).

If $S$ is smooth and strictly convex, the map

$$
n: x \rightarrow n(x)
$$

is a homeomorphism of $S$ onto $S^{d-1}$.

If $I \subset S$, then $n(I)$ is the subset of $S^{d-1}$ consisting of the normal images of the points of $I$.

We now give two definitions that we need to prove our result.

Definition 1. We say that property " $P$ " holds for a convex surface $S$ surrounding $C$ if

(a) $S \cap$ bd $C=I$ is not empty, and

(b) no open half-sphere of $S^{d-1}$ contains $n(I)$, where $n$ is the homeomorphism of $S$ onto $S^{d-1}$, defined above.

Definition 2. A set $I$ of points of $S$ is said to be irreducible if

(i) no open half-sphere of $S^{d-1}$ contains $n(I)$, but

(ii) for each proper subset $I^{\prime}$ of $I$ there is an open half-sphere of $S^{d-1}$ containing $n\left(I^{\prime}\right)$.

We mention that, for irreducible $I, \operatorname{card}(I) \leq d+1$ and that if $\operatorname{card}(I) \leq d$, then there is a hyperplane through the center of $S^{d-1}$ which contains $n(I)$. We remark that by condition (i) such a hyperplane exists also for $\operatorname{card}(I)=d$

\section{Preliminaries}

First we remark that, given a convex body $C$, the existence of an element of $\mathscr{T}$, whose convex hull includes $C$ and is of minimal volume, is easy to prove. The above element will always be denoted by $S(C)$. Next, with a proof analogous to that given by Bonnesen [1] for the sphere, it is possible to show that property " $P$ " holds for $S(C)$.

We also need the following lemmas.

Lemma 1. The $\mathscr{S}$-circumscribed surface of $C$ is unique for each convex body $C$. 
Lemma 2. If $C_{1}, C_{2}, \ldots \in \mathscr{C}$ and $\lim _{n \rightarrow \infty} C_{n}=C$ then

$$
\lim _{n \rightarrow \infty} S\left(C_{n}\right)=S(C)
$$

Lemra 3. Let $I$ be an irreducible subset of $S$ of cardinality $d+1$. Then $S$ is $\mathscr{S}$-circumscribed about conv $I$.

The proofs of these three lemmas are straightforward.

Lemma 4. Let $I=\left\{p_{1}, p_{2}, \ldots, p_{k}\right\}$ be an irreducible subset of $S$, where $k \leq d$. Then there is an irreducible subset of $S$ arbitrarily close to $I$ and consisting of $k+1$ points.

Proof. Let $n\left(p_{i}\right)(i=1,2, \ldots, k)$ be the normal image of the point $p_{i}(i=1,2, \ldots, k)$, and, for each $i$, let $I_{i}$ be the set

$$
I_{i}=\left\{p_{1}, p_{2}, \ldots, p_{i-1}, p_{i+1}, \ldots, p_{k}\right\}
$$

Choose a hyperplane $\Pi_{i}$ through the center $c$ of $S^{d-1}$ such that the points of $n\left(I_{i}\right)$ belong to the same open half-space bounded by $\Pi_{i}$. The hyperplanes $\Pi_{1}$, $\Pi_{2}, \ldots, \Pi_{k-1}$ divide $S^{d-1}$ into regions; we denote by $\Sigma$ the region containing $n\left(p_{k}\right)$. Now let $\Pi$ be a hyperplane containing the points $n\left(p_{i}\right)(i=1,2, \ldots, k)$ and also the center $c$. Next we choose two elements $n\left(q_{1}\right)$ and $n\left(q_{2}\right)$ in $\Sigma$ which are coplanar with $n\left(p_{k}\right)$ and $c$, are close to $n\left(p_{k}\right)$, and do not belong to the same open half-sphere bounded by $\Pi$.

Since it is easy to prove that the set $\left\{p_{1}, p_{2}, \ldots, p_{k-1}, q_{1}, q_{2}\right\}$ is an irreducible set of $S$, we show only, e.g., that $I^{\prime}=\left\{p_{1}, p_{2}, \ldots, p_{k-1}, q_{2}\right\}$ fulfills condition (ii). To this end we take a hyperplane through $n\left(p_{1}\right), n\left(p_{2}\right), \ldots, n\left(p_{k-1}\right), n\left(q_{2}\right)$ but not through the center $c$. This is possible also for $k=d$ since $n\left(q_{2}\right) \notin \Pi$. Then the hyperplane parallel to it and through the center $c$ determines an open half-sphere containing $n\left(I^{\prime}\right)$.

Choosing the points $n\left(q_{1}\right)$ and $n\left(q_{2}\right)$ close to $n\left(p_{k}\right)$ implies that $q_{1}$ and $q_{2}$ are also close to $p_{k}$.

\section{Main Result}

Theorem. Most convex bodies $C$ in $\mathscr{C}$ have precisely $d+1$ points in common with the $\mathscr{S}$-circumscribed surface of $C$. These points form an irreducible subset of $S(C)$.

Proof. Let $\mathscr{B}$ be the set of convex bodies $C \in \mathscr{C}$ such that bd $C \cap S(C)$ has cardinality $d+1$ and is an irreducible subset of $S(C)$. We show that $\mathscr{B}$ is dense in $\mathscr{C}$.

Let $D$ be an element of $\mathscr{C}$. Property " $P$ " holds for $S(D)$. If bd $D \cap S(D)$ contains an irreducible subset $I$ of $S(D)$ of cardinality $d+1$ we consider

$$
D(\lambda)=(1-\lambda) D+\lambda \text { conv } I \quad \text { with } \lambda \in(0,1) .
$$


From Lemma 3 we have

$$
S(\operatorname{conv} I)=S(D)
$$

Therefore, since $S(D(\lambda))=S(D)$ and bd $D(\lambda) \cap S(D)=I$, it follows that

$$
D(\lambda) \in \mathscr{B} \text {. }
$$

Then choosing $\lambda$ sufficiently small we have that $D(\lambda)$ is sufficiently close to $D$.

On the other hand if bd $D \cap S(D)$ contains an irreducible set $I$ of $S(D)$ of fewer than $d+1$ points, we can construct, as in Lemma 4, an irreducible set $J \subset S(D)$ of cardinality $d+1$ and close to $I$. Next we consider

$$
D(\lambda)=(1-\lambda) \operatorname{conv}(D \cup J)+\operatorname{conv} J, \quad \text { where } \quad \lambda \in(0,1) .
$$

Since

$$
S(D(\lambda))=S(D)
$$

and

$$
\text { bd } D(\lambda) \cap S(D)=J
$$

we have that $D(\lambda) \in \mathscr{B}$ and that $D(\lambda)$ can be constructed sufficiently close to $D$.

Now let

$$
\begin{aligned}
\mathscr{C}(i)= & \{C: C \in \mathscr{C} \text { such that bd } C \cap S(C) \text { contains more than } d+1 \text { points } \\
& \text { with mutual distances } \geq 1 / i\}
\end{aligned}
$$

and

$$
\begin{aligned}
\mathscr{C}(0)= & \{C: C \in \mathscr{C} \text { such that bd } C \cap S(C) \text { contains an irreducible subset } \\
& \text { of } S(C) \text { of fewer than } d+1 \text { points }\} .
\end{aligned}
$$

The proofs that $\mathscr{C}(i)$ and $\mathscr{C}(0)$ are closed are routine (see [3], [4], and [6]). Obviously,

$$
\mathscr{B}=\mathscr{C} \backslash\left(\mathscr{C}(0) \cup \bigcup_{i=1}^{\infty} \mathscr{C}(i)\right)
$$

This concludes the proof of the theorem.

\section{References}

1. T. Bonnesen, Les problèmes des isopérimètres et des isépiphanes, Gauthier-Villars, Paris, 1929.

2. P. Gruber, Results of Baire category type in convexity, Discrete Geometry and Convexity, Annals of the New York Academy of Sciences, vol. 440 (1985), pp. 163-169. 
3. P. Gruber, Minimal ellipsoids and their duals, Rend. Circ. Mat. Palermo (2) XXXVII (1988), 35-64

4. T. Zamfirescu, Inscribed and circumscribed circles to convex curves, Proc. Amer. Math. Soc. 80 (1980), 455-457.

5. T. Zamfirescu, Using Baire categories in geometry, Rend. Sem. Mat. Univ. Politec. Torino 43(1) (1985), 67-88.

6. A. Zucco, The minimal shell of a typical convex body, Proc. Amer. Math. Soc. 109 (1990), 797-802.

Received March 22, 1990, and in revised form June 19, 1990. 